\title{
ON THE COHOMOLOGY CHERN CLASSES OF THE $K$-THEORY CHERN CLASSES
}

\author{
MI-SOO BAE SMITH AND LARRY SMITH
}

Abstract. Let $\xi$ be a vector bundle over a finite complex and $\gamma^{i} \xi$ its $i$ th- $K$ theory Chern class. We first show that

$$
c_{n} \gamma^{i} \xi=(i-1) ! S(n, i) c_{n} \xi+\text { decomposables, }
$$

where $S(n, i)$ is a Stirling number of the second kind. We apply this result to show that certain multiples of the $e$-invariant of a map $S^{2 m-1} \rightarrow S^{2 n}$ must always be integral.

1. On Chern classes of Chern classes. Let $X$ be a finite complex and $\xi \in \tilde{K}(X)$. Then $\xi$ has $K$-theory Chern classes [6] $\gamma^{i \xi} \in K^{2 i}(X)$

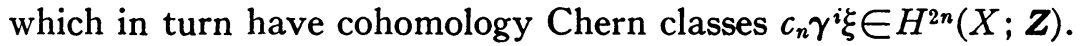

Our objective is to study the problem of computing $c_{n} \gamma^{i \xi}$ as a function of $c_{j} \xi$. Contemplation of the universal example shows that there must be a universal formula expressing $c_{n} \gamma^{i \xi}$ as a polynomial in $c_{0} \xi$, $\cdots, c_{n} \xi$. As a step towards computing this polynomial we shall establish:

Theorem 1.1. Let $X$ be a finite complex and $\xi$ a vector bundle over $X$. Then we have

$$
c_{n} \gamma^{i} \xi=(i-1) ! S(n, i) c_{n}(\xi)+\text { decomposables. }
$$

(Here $S(n, i)$ is a Stirling number of the second kind. See the appendix for information about Stirling numbers.)

PROOF. It will clearly suffice to check the assertion in the universal example, i.e., with $X$ a giant skeleton of $B U$ and $\xi$ the restriction of the canonical bundle. Let

$$
\phi_{n}: S^{2 n} \rightarrow B U
$$

be a representative of a generator of $\pi_{2 n}(B U) \cong Z$. Recall [2] that

$$
c_{n}\left(\xi_{n}\right)=(n-1) ! \sigma_{n}
$$

where $\xi_{n}$ is the bundle induced by $\phi_{n}$ over $S^{2 n}$ and $\sigma_{n} \in H^{2 n}\left(S^{2 n} ; \boldsymbol{Z}\right)$ is the canonical generator.

Now recall that we have shown $[5,2.4,2.5]$ that

$$
\operatorname{ch}^{2 n} \gamma^{i} \xi_{n}=(i-1) ! S(n, i) \sigma_{n} .
$$

Received by the editors December 30, 1969.

AMS 1969 subject classifications. Primary 5732, 5540; Secondary 5530, 1005.

$K e y$ words and phrases. $K$-theory, characteristic classes, Chern classes, $e$-invariant, Stirling numbers. 
However we also have the universal formula

$$
\operatorname{ch}^{2 n} \eta=(1 / n !)\left(n c_{n}(\eta)+\text { decomposables }\right)
$$

for any bundle $\eta$ over a finite complex. Since decomposables vanish in a sphere this specializes to

$$
\operatorname{ch}^{2 n} \gamma^{i \xi_{n}}=(1 /(n-1) !) c_{n} \gamma^{i \xi_{n}} .
$$

Equating these two expressions for $\operatorname{ch}^{2 n} \gamma^{i} \xi_{n}$ leads to the formula

$$
c_{n} \gamma^{i} \xi_{n}=(i-1) ! S(n, i)(n-1) ! \sigma_{n}
$$

and recalling that $(n-1) ! \sigma_{n}=c_{n} \xi_{n}$ we obtain

$$
c_{n} \gamma^{i} \xi_{n}=(i-1) ! S(n, i) c_{n} \xi_{n} .
$$

If we let $\theta$ denote the universal bundle over $B U$ then we have the universal formula $c_{n} \gamma^{i} \theta=A_{n, i} c_{n} \theta+$ decomposables. Applying $\phi_{n}^{*}$ gives $c_{n} \gamma^{i} \xi_{n}=A_{n, i} c_{n} \xi_{n}$ and the result follows.

CoROLlaRY 1.2. Let $X$ be a finite complex and $\xi$ a vector bundle over $X$. Then we have

$$
\operatorname{ch}^{2 n} \gamma^{i \xi}=(i-1) ! S(n, i) \operatorname{ch}^{2 n} \xi+\text { decomposables. }
$$

PROoF. This is implicit in the proof of (1.1) above and in addition follows easily from it. $\square$

CoROllary 1.3. Let $X$ be a finite complex and $\xi$ a vector bundle over $X$. Suppose that all products are zero in $\widetilde{H}^{*}(X ; Q)$, for example $X$ is a suspension. Then

$$
\operatorname{ch}^{2 n} \gamma^{i \xi}=(i-1) ! S(n, i) \operatorname{ch}^{2 n} \xi .
$$

If moreover all products are zero in $\tilde{H}^{*}(X ; Z)$ then

$$
c_{n} \gamma^{i \xi}=(i-1) ! S(n, i) c_{n} \xi \text {. }
$$

2. On the unstable $e$-invariant. Suppose given $f: S^{2 m-1} \rightarrow S^{2 n}$. Form the cofibration

$$
S_{i}^{2 n} \underset{i}{\hookrightarrow} S^{2 n} \cup_{f} e^{2 m} \underset{j}{\rightarrow} S^{2 m}
$$

yielding the exact sequence

$$
0 \leftarrow \tilde{K}\left(S^{2 n}\right) \stackrel{i^{*}}{\leftarrow} \tilde{K}\left(S^{2 n} \cup_{f} e^{2 m}\right) \stackrel{j^{*}}{\leftarrow} \tilde{K}\left(S^{2 m}\right) \leftarrow 0 .
$$

Choose generators $\lambda, \mu \in K\left(S^{2 n} \cup_{f} e^{2 m}\right)$ such that $j^{*} \xi_{m}=\lambda, i^{*} \mu=\xi_{n}$, where for any integer $k, \xi_{k} \in \tilde{K}\left(S^{2 k}\right) \cong \boldsymbol{Z}$ is the canonical generator. We then have 


$$
\operatorname{ch} \lambda=\sigma_{m}, \quad \operatorname{ch} \mu=e(f) \sigma_{m}+\sigma_{n}: \quad e(f) \in Q,
$$

where as previously $\sigma_{k} \in H^{2 k}\left(S^{2 k} ; \boldsymbol{Z}\right) \cong \boldsymbol{Z}$ is the canonical generator. The rational number $e(f)$ is of course the $e$-invariant of $f$ and its residue class in $Q / Z$ is independent of the choice of $\lambda, \mu$ [1], [7]. Our objective is to show that certain multiples of $e(f)$ must always be integral. To this end we introduce:

Definition. Let $m, n, k \in \boldsymbol{Z}^{+}$, and set

$$
g(m, n)=\underset{k>1}{\text { g.c.d. }}\left\{k^{m}-k^{n}\right\} .
$$

In the appendix we will establish that

$$
g(m, n)=\underset{1<\boldsymbol{k}<1+\max (m+1, n+1)}{\text { g.c.d. }}\{(k-1) ![S(m+1, k)-S(n+1, k)]\}
$$

where $m, n, k \in Z^{+}$.

The main result of this section is the following.

ThEOREM 2.1. Let $f: S^{2 m-1} \rightarrow S^{2 n}$ and assume that $m \neq 2 n$. Then $g(m-1, n-1) e(f) \in \boldsymbol{Z}$.

This result is among results originally obtained by P. Hoffman in [3] extending a stable result of Adams [1] and Toda [7].

Proof of 2.1. Let us continue to employ the notations of the previous discussion. Then on general grounds we have

$$
\gamma^{i} \mu=r_{i} \mu+s_{i} \lambda: \quad r_{i}, s_{i} \in Z .
$$

Moreover as we are assuming $m \neq 2 n$ we obtain in view of (1.3)

$$
\begin{aligned}
& \operatorname{ch}^{2 m} \gamma^{i} \mu=(i-1) ! S(m, i) \operatorname{ch}^{2 m} \mu=(i-1) ! S(m, i) e(f) \sigma_{m}, \\
& \operatorname{ch}^{2 n} \gamma^{i} \mu=(i-1) ! S(n, i) \operatorname{ch}^{2 n} \mu=(i-1) ! S(n, i) \sigma_{n},
\end{aligned}
$$

while from (A) we obtain

$$
\begin{aligned}
& \operatorname{ch}^{2 m} \gamma^{i} \mu=r_{i} \operatorname{ch}^{2 m} \mu+s_{i} \operatorname{ch}^{2 m} \lambda=r_{i} e(f) \sigma_{m}+s_{i} \sigma_{m}, \\
& \operatorname{ch}^{2 n} \gamma^{i} \mu=r_{i} \operatorname{ch}^{2 n} \mu+s_{i} \operatorname{ch}^{2 n} \lambda=r_{i} \sigma_{n} .
\end{aligned}
$$

Equating $(\mathrm{C})$ and $(\mathrm{E})$ leads to

$$
r_{i}=(i-1) ! S(n, i),
$$

while equating (B) and (D) and employing $(F)$ gives

$$
(i-1) ![S(m, i)-S(n, i)] e(f)=s_{i} \in \boldsymbol{Z} .
$$

Since $i$ is arbitrary we conclude that

$$
\text { g.c.d. }\{(i-1) ![S(m, i)-S(n, i)]\} e(f) \in \boldsymbol{Z} \text {. }
$$


But $g(m-1, n-1)=$ g.c.d. $\{(i-1) ![S(m, i)-S(n, i)]\}$ by the theorem from the appendix previously quoted.

Appendix: On Stirling numbers. Let us first recall one definition of the Stirling numbers of the second kind [4, II.7].

Definition. Let $n, k$ be positive integers. Then

$$
S(n, k)=\frac{(-1)^{k}}{k !} \sum_{j=0}^{k}(-1)^{j}\left(\begin{array}{l}
k \\
j
\end{array}\right) j^{n} .
$$

Note that

$$
S(n, 1)=1=S(n, n), \quad S(n, k)=0: \quad k>n,
$$

and

$$
S(n+1, k)=S(n, k-1)+k S(n, k),
$$

and so from the two conditions above we obtain $S(n, k) \in Z^{+} ; k$ $=1, \cdots, n$.

These numbers have a long history and many useful properties, see for example [4], [5].

The formula

$$
(-1)^{k} k ! S(n, k)=\sum_{j=0}^{k}(-1)^{j}\left(\begin{array}{l}
k \\
j
\end{array}\right) j^{n}
$$

may be inverted to yield a formula expressing $k^{n}$ as a function of $S(n, j), j=1, \cdots, n$. (This formula is of ten used to define the Stirling numbers.) The procedure runs as follows.

Lemma 1. Let $m, n \in \boldsymbol{Z}^{+}$, then

$$
\sum_{j=0}^{m}(-1)^{j}\left(\begin{array}{l}
m \\
j
\end{array}\right)(-1)^{n}\left(\begin{array}{l}
j \\
n
\end{array}\right)=\delta_{m n}
$$

whenever $n \leqq m$.

Proof. Start from the identity $t^{m}=(1-(1-t))^{m}$ and apply the binomial theorem twice to yield

$$
t^{m}=\sum_{j=0}^{m}(-1)^{j}\left(\begin{array}{c}
m \\
j
\end{array}\right)(1-t)^{j}=\sum_{j, n=0}^{m}(-1)^{j}\left(\begin{array}{l}
m \\
j
\end{array}\right)(-1)^{n}\left(\begin{array}{l}
j \\
n
\end{array}\right) t^{n}
$$

and the result follows from equating coefficients. $\square$

Return now to the formula

$$
(-1)^{k} k ! S(n, k)=\sum_{j=0}^{k}(-1)^{j}\left(\begin{array}{l}
k \\
j
\end{array}\right) j^{n}
$$


Multiplying by $(-1)^{k}\left(\begin{array}{l}i \\ k\end{array}\right)$ and summing from $k=0$ to $k=i$ we get

$$
\sum_{k=0}^{i} k !\left(\begin{array}{l}
i \\
k
\end{array}\right) S(n, k)=\sum_{k=0}^{i} \sum_{j=0}^{k}(-1)^{k}\left(\begin{array}{l}
i \\
k
\end{array}\right)(-1)^{j}\left(\begin{array}{l}
k \\
j
\end{array}\right) j^{n} .
$$

Interchanging the order of summation and applying Lemma 1 we obtain

$$
\sum_{k=0}^{i} k !\left(\begin{array}{l}
i \\
k
\end{array}\right) S(n, k)=\sum_{j=0}^{k} \delta_{i j} j^{n}=i^{n}
$$

Hence we have established:

Proposition 2. We have the formula $i^{n}=\sum_{x=0}^{i} k !\left(\begin{array}{l}i \\ k\end{array}\right) S(n, k)$, for all $n, i \in \boldsymbol{Z}^{+}$.

Corollary 3. Let $n, m, k$ be positive integers. Then we have the formulas

$$
k^{m}-k^{n}=\sum_{i=0}^{k} i !\left(\begin{array}{l}
k \\
i
\end{array}\right)[S(m, i)-S(n, i)]
$$

and

$$
(-1)^{k} k ![S(m, k)-S(n, k)]=\sum_{i=0}^{k}(-1)^{i}\left(\begin{array}{l}
k \\
i
\end{array}\right)\left(i^{m}-i^{n}\right) .
$$

Proof. The first assertion follows from Proposition 2 and the second from the definitions. $\square$

Corollary 4. Let $n, m \in Z^{+}$, then

$$
g(m, n)=\underset{1<\boldsymbol{k}<1+\max (n, m)}{\text { g.c.d. }}\{k ![S(m, k)-S(n, k)]\} .
$$

Proof. Immediate from Corollary 3 and the fact that $S(r, s)=0$ for $s>r$.

More generally than Corollary 4 we have:

Proposition 5. Let $m, n \in Z^{+}$. Then

$$
(-1)^{k}(k-1) ![S(m, k)-S(n, k)]=\sum_{i=1}^{k}(-1)^{i}\left(\begin{array}{l}
k-1 \\
i-1
\end{array}\right)\left(i^{m-1}-i^{n-1}\right),
$$

$k \in Z^{+}$

Proof. The result takes almost as long to state as to prove.

Start with the formula 


$$
(-1)^{k} k ![S(m, k)-S(n, k)]=\sum(-1)^{i}\left(\begin{array}{l}
k \\
i
\end{array}\right)\left(i^{m}-i^{n}\right) .
$$

Using the binomial identity

$$
\left(\begin{array}{l}
k \\
i
\end{array}\right) \frac{1}{k}=\left(\begin{array}{c}
k-1 \\
i-1
\end{array}\right) \frac{1}{i}
$$

rewrite it as

$$
\begin{aligned}
(-1)^{k}(k-1) ![S(m, k)-S(n, k)] & =\sum(-1)^{i}\left(\begin{array}{l}
k-1 \\
i-1
\end{array}\right) \frac{1}{i}\left(i^{m}-i^{n}\right) \\
& =\sum(-1)^{i}\left(\begin{array}{l}
k-1 \\
i-1
\end{array}\right)\left(i^{m-1}-i^{n-1}\right)
\end{aligned}
$$

which is the desired result.

Applying inversion and what not we obtain:

$$
k^{m-1}-k^{n-1}=\sum_{i=1}^{k}(k-1) !\left(\begin{array}{c}
k-1 \\
i-1
\end{array}\right)[S(m, i)-S(n, i)]
$$

and hence we get:

Corollary 6. Let $m, n \in \boldsymbol{Z}^{+}$, then

$$
g(m-1, n-1)=\underset{1<\boldsymbol{k}<1+\max (n, m)}{\text { g.c.d. }}\{(k-1) !(S(m, k)-S(n, k))\},
$$

\section{REFERENCES}

1. J. F. Adams, On the groups $J(X)$. IV, Topology 5 (1966), 21-71. MR 33 \#6628.

2. H. Cartan and J. C. Moore, Periodicite des groupes d'homotopie stables des groupes classiques, d'après Bott, Séminaire H. Cartan, Secrétariat mathématique, Paris, 1961. MR 28 \#1092.

3. P. Hoffman, On the unstable e-invariant, Topology 4 (1965), 343-349. MR 33 \#6629.

4. J. Riordan, An introduction to combinatorial analysis, Wiley, New York, 1958. MR $20 \# 3077$.

5. L. Smith, On the $K$-theory characteristic numbers of almost complex manifolds with framed boundaries, University of Virginia, Charlottesville, Va., 1969; Topology (to appear).

6. R. E. Stong, Notes on cobordism theory, Princeton University Lecture Notes, Princeton Univ. Press, Princeton, N.J., 1968.

7. H. Toda, $A$ survey of homotopy theory, Sugaku 15 (1963/64), 141-155. (Japanese) MR 29 \#6494.

University of Virginia, Charlottesville, Virginia 22901 